\title{
Energy Efficient Node Deployment for Heterogeneous Wireless Sensor Networks
}

\author{
Shahzad Hassan ${ }^{\text {a }}$, Shahida Khanam ${ }^{\text {a }}$, Maria Ahmad ${ }^{\text {a }}$ Imtiaz Alam ${ }^{\text {b }}$ \\ ${ }^{a}$ Computer Engineering Department, Bahria University, Pakistan \\ ${ }^{\mathrm{b}}$ Electrical Engineering Department, Barhria University \\ * corresponding author: shassan.buic@ bahria.edu.pk
}

\begin{abstract}
Wireless Sensor nodes are power-constrained devices and the lifetime of the nodes is significantly influenced by the deployment of the nodes. In the appropriate deployment of the nodes leads to inefficient network energy utilization which results in a minimized network lifetime. To enhance the network lifetime within the limited battery power, hierarchical heterogeneous routing has been proposed by various researchers and have significantly prolonged the network lifetime. In heterogeneous wireless sensor networks, the researchers have addressed mainly energy efficiency of the network but node deployment has been rarely addressed. Node deployment is a critical issue in heterogeneous wireless sensor networks since the nodes in these networks are energized with different energy levels. The simulation results obtained proved that the appropriate deployment of nodes can lead to a substantial amount of energy conservation.
\end{abstract}

Keywords- WSN; Heterogeneous; nodes; deployment; energy

Date Received: $04-02-2021$

Date Accepted: 09-03-2021

Date Published: 08-06-2021

\section{INTRODUCTION}

$\mathrm{W}$ ireless sensor networks (WSNs) are composed of

randomly deployed sensor nodes for sensing and data gathering within its sensing radius. Node deployment is a vital issue in WSN design. Though, deployment of sensors nodes is an essential aspect in WSNs as it significantly affects the performance of the network, such as connectivity [1], network lifetime [2], coverage area [3] and energy efficiency [4]. In WSNs, nodes can be organized either randomly or in a predetermined way [5]. In random deployment sensor nodes are scattered randomly in a harsh or hostile phenomenon where human intervention is impossible or dangerous, while in predetermined placement the nodes are deployed in a specified site. Since the WSN is energy-constrained devices and it is desirable to optimize the energy consumption of the nodes to enhance the network lifetime and poor deployment of nodes will degrade the network performance.

Due to the energy conservation issue, hierarchal routing protocols have been proposed by various researchers [4]. Normally the hierarchal routing protocols have been divided into two group homogeneous clustering and heterogeneous clustering. In homogenous clustering, the network is equipped with identical nodes having identical energy levels while in a heterogeneous clustering network is composed of nodes having different energy levels. In the clustering technique, the nodes are partitioned into groups known as clusters. Commonly the cluster is comprised of three main elements. Cluster Heads (CHs), Cluster Member Nodes (CMs), and the base station (BS) [6]. In general, one $\mathrm{CH}$ is selected from the clusters and the remaining cluster members connect with the cluster head based on the minimum distance from cluster head. The cluster members (CMs) communicate only with the cluster head and forward the sensed data to $\mathrm{CH}$, due to the random placement of the nodes, sometimes the nodes are closely deployed to each other and their sensing region overlaps with other which results in highly correlated data. The cluster head then performs data aggregation and eliminates the correlated data and data fusion on the data obtained by the cluster members and transmits the fused data to the base station for further end-user processing [2]. The CHs selection criteria are varying from protocol to protocol but mostly it is based on nodes energy level, distance from the BS, residual energy, etc.

In heterogeneous clustering, the nodes having high energy levels are selected as cluster heads. Since heterogeneous wireless sensor networks (HWSN) are comprised of multi-tier nodes having different energy levels, the random deployment of nodes is not a feasible solution [4]. There are numerous objectives for using clusters in WSNs, including network connectivity, fault tolerance and load balancing [5].

However, the node deployment significance on the lifetime of the nodes in a randomly deployed network has been mainly unaddressed in clustering protocols. Subsequently in clustering protocols long haul transmission is involved, the inappropriate deployment of the nodes will affect the network coverage, transmission rate and as well as lifetime of the overall network, making the nodes deployment vital problems in clustering protocols. 


\section{CLUSTERING PROCESS}

Designing of the clustered network is on the most crucial step for effectively utilizing the network energy. During the designing issue, certain aspects should be considered; like optimal cluster head size, $\mathrm{CHs}$ selection criteria, etc. The clustering establishment process can be divided into three main phases' i.e. (i) cluster head selection, (ii) cluster formation and (iii) data transmission phase. Various research has been proposed to implement each of these phases [6].

\section{A. Cluster Head Selection}

$\mathrm{CH}$ selection can be classified into three categories, centralization by the BS, decentralization by the nodes or hybrid selection and some by the nodes themselves. $\mathrm{CH}$ selection is a foremost challenge to prolong the network's lifetime. The $\mathrm{CH}$ is selected among the existing sensor nodes and the selection measures of $\mathrm{CH}$ vary in the suggested research but the key intention of these studies is to decrease the energy intake and prolong the network life-span [7]. To minimize the routing complexity and make the network more energy efficient it is necessary to decide the optimum number of $\mathrm{CH}$, which will minimize the overhead while maintaining the network connectivity in case of topology changes occurs. Researchers have proposed different mechanisms to select cluster head but still it open research problem.

HSWSN are generally two-level protocols equipped with normal nodes having initial energy of E0 and $\mathrm{m}$ advance nodes having extra energy $\alpha$ as related to normal nodes. The total energy of the network is increased to " $(1+\mathrm{m} . \alpha)$ " times. So, the whole initial energy of the network develops to:

$\mathrm{E}_{\mathrm{TOT}}=\mathrm{nE}_{0}(1+\mathrm{m} \alpha)$

The probability of normal nodes $\left(\mathrm{P}_{\mathrm{NS}}\right)$ and advance nodes $\left(\mathrm{P}_{\mathrm{AS}}\right)$ to be elected as $\mathrm{CHs}$ becomes [8]:

$$
\begin{aligned}
\mathrm{P}_{\mathrm{NS}} & =\frac{\mathrm{P}_{\mathrm{opt}}}{(1+\alpha * \mathrm{~m})} \\
\mathrm{P}_{\mathrm{AS}} & =\frac{\mathrm{P}_{\mathrm{opt}}}{(1+\alpha * \mathrm{~m})} *(1+\alpha)
\end{aligned}
$$

The CHs is election is based on threshold value which is set between 0 and1. If the computed value is higher than the previous one, $\mathrm{CHs}$ are selected. The threshold value for normal nodes and advance nodes is:

$$
\begin{aligned}
& \mathrm{T}_{\left(\mathrm{S}_{\mathrm{N} S}\right)}=\left\{\begin{array}{cc}
\frac{\mathrm{P}_{\mathrm{NS}}}{1-\mathrm{P}_{\mathrm{NS}} *\left(\mathrm{r} * \bmod \left(\frac{1}{\mathrm{P}_{\mathrm{NS}}}\right)\right.} & \text { if } \mathrm{S}_{\mathrm{NS}} \in \mathrm{X}^{\prime} \\
0 & \text { otherwise }
\end{array}\right. \\
& =\left\{\begin{array}{cc}
\frac{\mathrm{P}_{\mathrm{AS}}}{1-\mathrm{P}_{\mathrm{AS} *} *\left(\mathrm{r} * \bmod \left(\frac{1}{\mathrm{P}_{\mathrm{AS}}}\right)\right.} & \text { if } \mathrm{S}_{\mathrm{AS}} \in \mathrm{X}^{\prime \prime} \\
0 & \text { Otherwise }
\end{array}\right.
\end{aligned}
$$

Where, $\mathrm{r}$ is the present round, $\mathrm{X}^{\prime}$ and $\mathrm{X}^{\prime \prime}$ are nodes that are not selected as $\mathrm{CH}$ with in the last round.

\section{B. Cluster Formation}

In cluster formation or set-up phase, the cluster formation takes place. The $\mathrm{CHs}$ declare their selection to network nodes by broadcasting advertisement messages and each network node responds by sending a join message to the $\mathrm{CH}$. For the $\mathrm{N}$ number of network nodes, the certain number of clusters are formed during each round. The energy consumed by the $\mathrm{CH}$ in a certain round is computed by the following equation [11].

$$
\begin{gathered}
\mathrm{E}_{\mathrm{CH}=} \mathrm{P} \cdot \mathrm{E}_{\mathrm{Ckt}}\left(\frac{\mathrm{n}}{\mathrm{C}}-1\right)+\mathrm{P} \cdot \mathrm{E}_{\mathrm{AD}} \frac{\mathrm{n}}{\mathrm{C}}+\mathrm{P} \cdot \mathrm{E}_{\mathrm{Ckt}} \\
+ \text { P. } \mathrm{E}_{\mathrm{fs}} \mathrm{d}_{\mathrm{TX}}^{2}
\end{gathered}
$$

Where $\mathrm{C}$ is the sum of clusters, $\mathrm{E}_{\mathrm{AD}}$ aggregated data and $\mathrm{d}_{\mathrm{TX}}$ is the distance amongst the $\mathrm{CH}$ and $\mathrm{BS}$. The energy utilized by a non- $\mathrm{CH}$ is as follow

$$
\mathrm{E}_{\mathrm{NCH}}=\left\{\begin{array}{l}
\mathrm{P} \cdot \mathrm{E}_{\mathrm{Ckt}}+\mathrm{P} \cdot \varepsilon_{\mathrm{fs}} \cdot \mathrm{d}_{\mathrm{CH}}^{2} \text { if } \mathrm{d}_{\mathrm{CH}}<\mathrm{d}_{\mathrm{BS}} \\
\mathrm{P} \cdot \mathrm{E}_{\mathrm{Ckt}}+\mathrm{P} \cdot \varepsilon_{\mathrm{mp}} \cdot \mathrm{d}_{B S}^{2} \text { if } \mathrm{d}_{\mathrm{CH}}>\mathrm{d}_{B S}
\end{array}\right.
$$

Where $\mathrm{d}_{\mathrm{CH}}$ is the distance between the member node and the $\mathrm{CH}$ and its average value can be computed by " $\mathrm{d}_{\mathrm{CH}} \mathrm{M} /(\sqrt{2} 2 \pi)$ ". $\mathrm{d}_{\mathrm{sk}}$ is the distance amongst the nearest node and the BS.

\section{Data Transmission}

In data transmission or steady-state Phase, the cluster member forwards sense data to $\mathrm{CHs}$, and the $\mathrm{CH}$ performs data aggregation and forwards it to the BS. The total energy consumed in the network is equal to

$$
E_{\text {TOT }}=P .\left(2 n E_{\text {Ckt }}+n E_{A D}+E_{f s}\left(C . d_{T X}^{2}+n \frac{M^{2}}{2 \pi C}\right)\right)
$$

\section{RELATED WORK}

Appendixes, In literature abundant work has been carried out on relay node placement [9], connectivity [10], prolonging the network life [4] and maximizing the coverage area [11] of the network.

Conferring to the availability of the monitored zone, there are two sensor placement approaches deterministic sensor deployment and random sensor deployment. Generally, patternbased lattice WSNs ensuing from deterministic sensor node placement provides improved connectivity and coverage, compared to random deployments [12].

In deterministic deployments, there is an alternative task that are heterogeneous node deployments. In [13], the author researched the energy-efficient area coverage issue by regularly deploying sensors with variable sensing and communication ranges and suggested new density control designs that considerably expand coverage using dual sensing nodes.

The writers of [14] used relay nodes and suggested a heterogeneous node deployment scheme (HNDS) to enhance balanced energy intake and network lifetime inhomogeneous sensor networks considering network coverage region. To balance the load and energy intake, a researcher in [15] proposed the node deployment approach in which nodes were deployed in ascending density near the base station (BS). Consequently, additional nodes were required for data forwarding. The reference [16] presents WSNs placement 
algorithm based on virtual force, this algorithm can rapidly and efficiently design optimization of nodes. The mobile solutions acquired by the shared positional affiliation among nodes but the sensor nodes density greater effects the mobile solutions and can't influence global optimization purposes. Reference [17] investigated the maximum coverage quality complications under the condition of restricted entire budget in an extensive variety of regional occurrence monitoring with WSNs, and then used the relationship of the heterogeneous sensor nodes which have varied confidence to decrease the number of nodes, and lastly put forward an estimated algorithm based on greedy routing for nodes placement optimization.

Random node deployment is the most frequently considered node deployment method in the HWSN [18]. Though, it is inefficient from an energy efficiency perception due to Nodes different energy levels. The unfeasibility usually arises in two sorts of condition, one where the number of nodes is vast, and the other when the network is comprised of heterogeneous nodes i.e. nodes having various energy levels. In these scenarios, the requirement of a well-designed node deployment algorithm is becoming viable to maximize the network lifetime. But all this prior research work ignores the placement of advance nodes which have a higher priority to be elected as cluster head. The node deployment issue in HWSN has been not addressed by the researchers. In this article, nodes are deployed in deterministic fashion in the hexagonal structure.

\section{HEX-SEP}

In section, we will describe the network model, energy model, and sensing and communication model, and considered for this research.

\section{A. Hexagonal Node Deployment}

The nodes deployment degrades the network performance and overall minimizes the network lifetime. Typical wireless sensor networks deployment schemes consider the following node deployment criteria.

\section{-Coverage}

- Connectivity

-Network lifetime

While deploying traditional wireless sensor networks coverage and maintain connectivity is always considered. In HWSN $100 \%$ connectivity is guaranteed because the nodes join their respective cluster head based on minimum distance but due to random deployment whole area coverage may be sometimes compromised. Since the HWSN's are comprised of nodes having different energy levels, proper consideration should be given to the node deployment for prolonging the network time. A regular hexagonal deployment is considered in this article, as a good deployment in WSNs, particularly for the coverage performance. Each of the sensor nodes is deployed in the center of the cells with a sensing radius $\mathrm{R}$.

\section{B. Network Model}

The network comprised of mainly three sorts of nodes, network nodes and the base station (BS) deployed in regular hexagonal cells. The benefit of deploying in hexagonal cells will eliminate the overlapping of the sensing radius of network nodes.

Further, the network's nodes are divided into in advance and normal nodes. The advance nodes are higher in energy as compared to normal nodes and the energy of the base station is set to infinity.

\section{Energy Model}

This research implements the energy model anticipated in [9] as shown in figure (1).

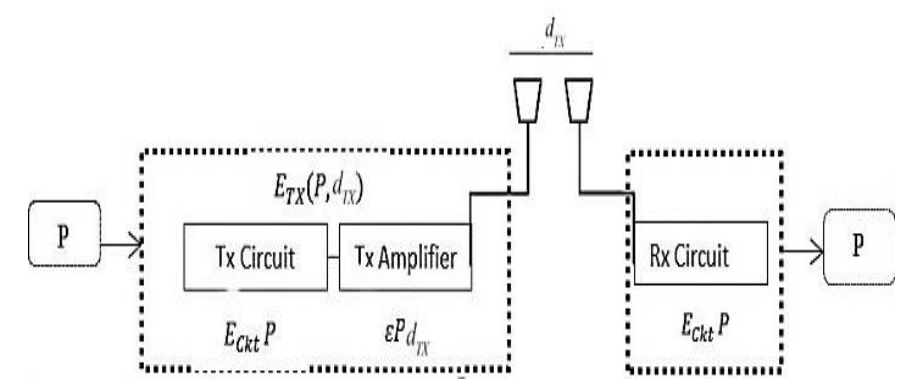

Figure 1: Energy Model

$$
\begin{aligned}
& E_{T x}\left(P, d_{T X}=\right. \\
& \left\{\begin{array}{l}
P . E_{C k t}+P . \varepsilon_{f s} \cdot d_{T X}^{2} \text { if } d_{T X}<d_{N S} \\
P . E_{C k t}+P . \varepsilon_{m p} \cdot d_{T X}^{4} \text { if } d_{T X} \geq d_{N S}
\end{array}\right.
\end{aligned}
$$

$\mathrm{E}_{\mathrm{TX}}\left(\mathrm{P}, \mathrm{d}_{T X}\right)$ is the energy exhausted by the network nodes in data transmission. $\mathrm{E}_{\mathrm{Ckt}}$ is the energy expended to make the circuit operational. $\mathrm{d}_{\mathrm{TX}}$ is the distance among the transmitter and receiver while the threshold distance between the base station and the network nodes is less than or equal to $d_{N S}$ By computing the two equations at $\mathrm{d}_{\mathrm{TX}}=\mathrm{d}_{\mathrm{NS}}$, we have " $\mathrm{d}_{\mathrm{NS}}=\sqrt{ } \varepsilon_{\mathrm{fs}} / \varepsilon_{\mathrm{mp}}$." The energy exhausted in receiving the data is equal to $E_{R X}=P$. $E_{C k t}$, where $E_{R X}$ is energy spent data reception.

\section{Sensing and Communication}

\section{E. Model}

The Sensing Radius, RRD of the sensor nodes can be expressed as the maximum distance in which the sensor nodes are capable to sense and gather the data. The sensing radius $\mathrm{R}_{\mathrm{RD}}$ is expected to be identical to the edge distance " $\mathrm{d}$ " of the hexagonal cellbased deployment pattern. The estimated length of a unit hexagon, d, can be calculated subsequently: Initial, the estimated area of a unit hexagon with measurement $d$ can be calculated by dividing the whole area having Radius $\mathrm{R}$, with the total number of hexagon cells, $\mathrm{k}$.

Area of unit square cell $=$ Complete Area of circular field $/$ Number of hexagonal cells in the area

For regular hexagonal deployment design, the estimated value of $\mathrm{k}$ is $(\mathrm{n} / 3-1)$. So, from the equation (2), we derive equation (3) for $R_{\text {Sen }}$, the sensing radius or the sensing range for the regular hexagonal-based deployment design [19]

$$
R_{\text {Sen }}=\frac{\pi R^{2}}{\left(\frac{3 \sqrt{3}}{2}\right)\left(\frac{n}{3}-1\right)}
$$




$$
R_{\text {Sen }}=\frac{\sqrt{6 \pi R^{2}}}{3 \sqrt{3 n}}
$$

\section{SIMULATIONS AND DISCUSSIONS}

In this section, we present some experimental results to verify the effectiveness of the proposed sensor deployment algorithm. The simulation is performed using MATLAB. To evaluate the performance of the network lifetime and energy efficiency have been considered as performance matrices. We have chosen the network first node and last node die and throughput as the yardsticks for measuring network lifetime defined in section. We have deployed 100 sensor nodes deployed at the center of each cell having a radius of four. The simulated cases describe the number of active and dead nodes, the number of packets transmitted to the base station by setting parameters for " $\mathrm{m}$ " which is a fraction of advanced nodes and $\alpha$ which is extra energy factor for advance nodes. Since in our case we are examining the energy heterogeneity we have considered only $\mathrm{m}=0.1$ and $\mathrm{a}=1$. Rests of the parameters are given in table 1 given below. We have performed independent simulations for each protocol.

Table 1: Parameters

\begin{tabular}{|l|l|}
\hline Parameter & Value \\
\hline Area & $100 * 100$ \\
\hline $\mathrm{n}$ & 100 \\
\hline $\mathrm{E}_{\mathrm{Ckt}}$ & $50 \mathrm{~nJ} / \mathrm{bit}$ \\
\hline $\mathrm{E}_{\mathrm{AD}}$ & $5 \mathrm{~nJ} / \mathrm{bit} / \mathrm{message}$ \\
\hline $\mathrm{E}_{0}$ & $0.5 \mathrm{~J}$ \\
\hline Packet Size & 4000 \\
\hline $\mathrm{P}_{\mathrm{opt}}$ & 0.1 \\
\hline$\varepsilon_{\mathrm{fs}}$ & $10 \mathrm{pJ} / \mathrm{bit} / \mathrm{m}^{\wedge} 2$ \\
\hline$\varepsilon_{\mathrm{mp}}$ & $0.0013 \mathrm{pJ} / \mathrm{bit} / \mathrm{m}^{\wedge} 4$ \\
\hline $\mathrm{d}_{\mathrm{NS}}$ & 87.70 \\
\hline
\end{tabular}

It can be observed from the figure $(2,3)$ that in terms of stable region, the HEX-SEP outperforms the SEP Protocol. The first node of HEX-SEP drains out its energy at 1240 round while in SEP 973 it is the reason is that SEP gives priority to advance nodes to be elected as cluster head as compared to normal nodes and a significant amount of energy is wasted in data aggregation process and moreover to inappropriate nodes deployment. It can

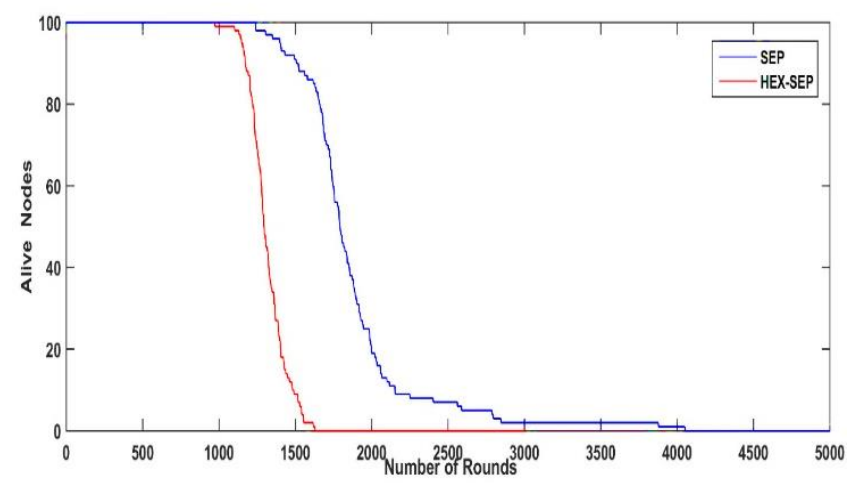

be seen in figure (3) the rate of energy depletion of advance nodes is slower in HEX-SEP as compared to SEP. This reason this that due to the non-over lapping sensing radius of nodes, a significant amount of energy is minimized which is wasted in the data aggregation process.

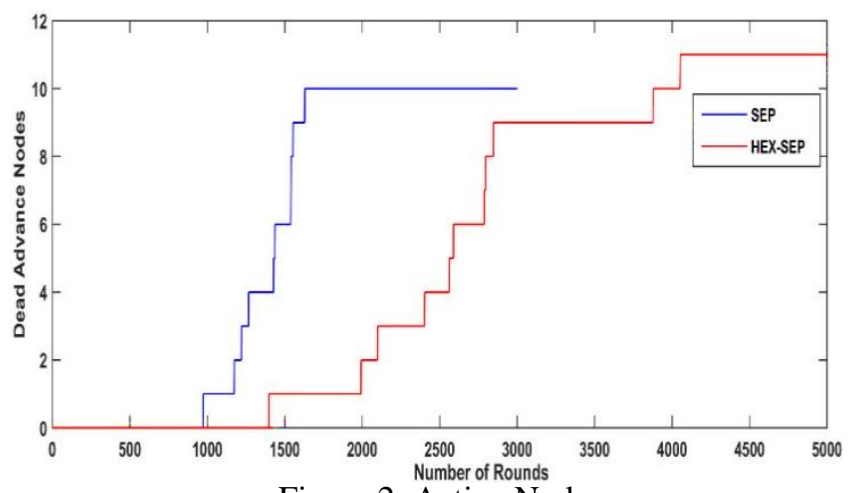

Figure 2: Active Nodes

Figure 3: Dead Advanced Nodes

Similar to the figure $(4,5)$ we examine that last node for HEX-SEP and SEP drains out their energy at 4052 and 1629 rounds respectively. The unstable region of HEX-SEP is significantly larger as compared to another SEP. Since the normal nodes are equipped with less energy as compared to advance nodes, when the normal nodes are selected as cluster heads their energy depletes at a faster rate and more overdue to the data aggregation process an excessive amount of energy is also wasted which can be seen in figure (5). In HEX-SEP the due to the non-overlapping radius the minimum amount of energy is utilized as compared to SEP.

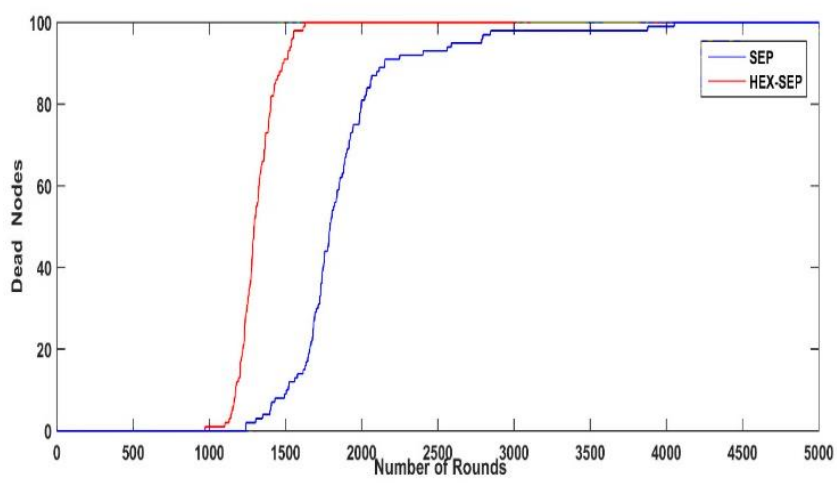

Figure 4: Dead Nodes

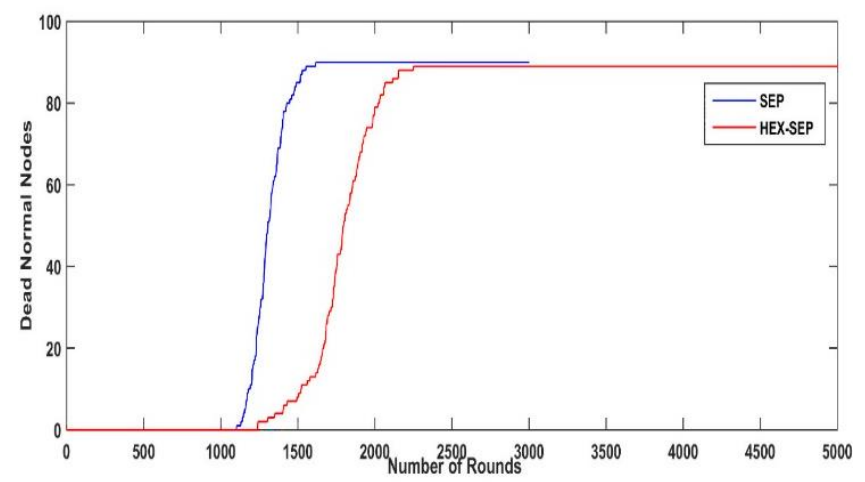

Figure 5: Dead Normal Nodes 
Figure (6) demonstrates the simulation results of throughput of by HEX-SEP and SEP. The throughput of the HEX-SEP is enormous as compared to SEP. Due to random deployment, sometimes the nodes sensing region overlaps with each other

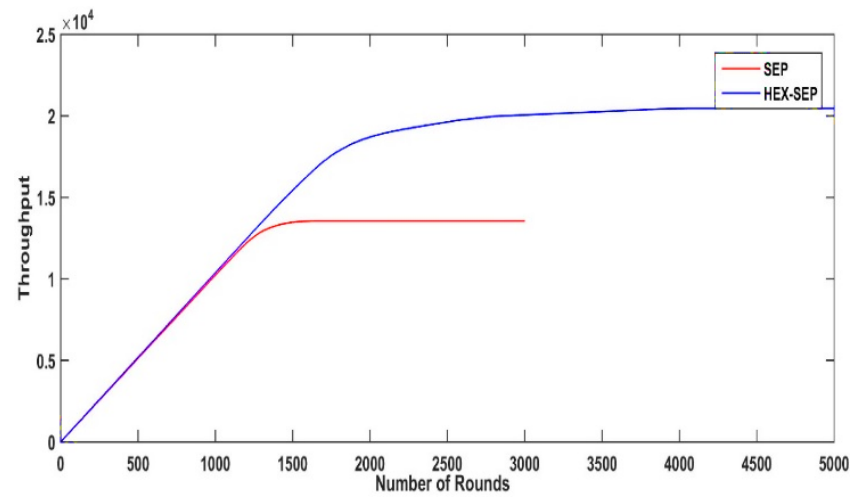

and high correlated data is gathered. The correlated data is eliminated by the data aggregation process. Due to the overlapping sensing regions, correlated data is collected which results in minimum data collection and moreover a significant amount of energy is also wasted in the data aggregation process. Figure 6: Throughput

\section{CONCLUSION}

Though the existing protocols have successfully enhanced the network lifetime still there are open issues that need to be addressed. Since HWSNs are comprised of multi-level nodes, greater consideration should be given to the node deployment strategies. Inappropriate node deployment results in a substantial amount of energy wastage in the data aggregation process which ultimately decreases the network lifetime.

\section{ACKNOWLEDGEMENTS}

These works were fully supported by HEC, Pakistan under the Start-Up Research Grant Program. Grant No.211286/SGRP/R\&D/HEC/2017

\section{REFERENCES}

[1] Jamal, N. and A., Amjad G. 2017. The Optimal Deployment, Coverage, and Connectivity Problems in Wireless Sensor Networks: Revisited IEEE Access 5, (August 2017), $18051 \quad$ - 18065. DOI: 10.1109/ACCESS.2017.2740382

[2] Padmalaya, N. and Anurag, D. 2016. A Fuzzy Logic-Based Clustering Algorithm for WSN to Extend the Network Lifetime. IEEE Sensors Journal 16,1 (Jan. 2016), 137 144. DOI: 10.1109/JSEN.2015.2472970

[3] Jiguo, Y. Shengli W. Xiuzhen, and C. Dongxiao, Y. 2017. Coverage Contribution Area Based k -Coverage for Wireless Sensor Networks IEEE Transactions on Vehicular Technology 66,9(Sep. 2017), 8510 - 8523. DOI: 10.1109/TVT.2017.2681692

[4] Aderohunmu, F.A. and Deng, J.D. 2010. An Enhanced Stabel Election Protocol (SEP) for Clustered Heterogeneous WSN. Discussion Paper Series, No.
2009/07. Department of Information Science, University of Ontago (2010)

[5] Ameer, A. A., and Mohamed Y.2007. A survey on clustering algorithms for wireless sensor networks, Network Coverage, and Routing Schemes for Wireless Sensor Networks, 30,14-15 (Oct. 2007), 2826-2841.DOI: 10.1016/j.comcom.2007.05.024

[6] S. Tanwara, N. Kumarb, J.P.C. Rodriguesc, "A systematic review on heterogeneous routing protocols for wireless sensor network", Journal of Network and Computer Applications, Vol.53, pp. 39-56, Jul. 2015

[7] D. Gupta and R. Verma, "An enhanced cluster-head selection scheme for distributed heterogeneous wireless sensor network", International Conference on Advances in Computing, Communications, and Informatics (2014)

[8] Smaragdakis, I. Matta and A. Bestavros, "SEP: A Stable Election Protocol for clustered heterogeneous wireless sensor networks", in Second International Workshop on Sensor and Actor Network Protocols and Applications, (2004)

[9] Kenan, X., Hossam, H., Glen, T. and Quanhong, W. 2010 Relay Node Deployment Strategies in Heterogeneous Wireless Sensor Networks, IEEE Transactions on Mobile Computing 9,12 (Feb. 2010),149-159. DOI: 10.1109/TMC.2009.105

[10] Chen Wang, Hongbo Jiang, Yan Dong. Connectivity-based Space Filling Curve Construction Algorithms in High Genus 3D Surface WSNs. ACM Transactions on Sensor Networks. 2016, 12(3): 22:1-22:29. Hamid, M. Kaveh, M., Amir, G. A. and Kamran, S.2017. Distributed Sensor Coordination Algorithms for Efficient Coverage in a Network of Heterogeneous Mobile Sensors, IEEE Transactions on Automatic Control, 62,11 (Nov. 2017). DOI: 10.1109/TAC.2017.2714102

[11] Halder, S., and Bit, S. D. 2014. Enhancement of wireless sensor network lifetime by deploying heterogeneous nodes. Journal of Network and Computer Applications, 38, (Feb. 2014) 106-124. DOI: /10.1016/j.jnca.2013.03.008

[12] Li, J., and Mohapatra, P. 2007. Analytical modeling and mitigation techniques for the energy hole problem in sensor networks, Pervasive and Mobile Computing, 3,3 (June 2007), 233-254.DOI: 10.1016/j.pmcj.2006.11.001

[13] Li, S., Ma, D., Li, Q., Zhang, J., and Zhang, X. 2013. Nodes Deployment Algorithm Based on Perceived Probability of Heterogeneous Wireless Sensor Advanced Mechatronic Systems (ICAMechS), 2013 International Conference on (Luoyang, China 25-27 Sept. 2013). 374-378. DOI: 10.1109/ISIT.2017.8007056

[14] Zhang, H.; Hou, J.C. Is Deterministic Deployment Worse than Random Deployment for Wireless Sensor Networks? In Proceedings of the 25th IEEE International Conference on Computer Communications (INFOCOM 2006), Barcelona, Spain, 23-29 April 2006.

[15] Zalyubovskiy, V.; Erzin, A.; Astrakov, S.; Choo, H. Energy-efficient Area Coverage by Sensors with 
Adjustable Ranges. Sensors 2009, 9, 2446-2460.

[CrossRef] [PubMed]

[16] Gao, J., Li, J., Cai, Z., and Gao, H.2015.Composite event coverage in wireless sensor networks with heterogeneous sensors[C] Computer Communications (INFOCOM), IEEE Conference on (Kowloon, Hong Kong, 26 April-1 May 2015).217-225.

DOI:

10.1109/INFOCOM.2015.7218385

[17] Ahmad, A., Slim, Z., Sofiène, A., and Nahi, K., Low-Cost Localization for Multihop Heterogeneous Wireless Sensor Networks 2016, IEEE Transactions on Wireless Communications 15,1 (Jan. 2016) 472 - 484. DOI: 10.1109/TWC.2015.2475255

[18] Yong, K., Chan, K., Dong, Y., Young, and Oh., Youn, H. 2012 Regular sensor deployment patterns for p-coverage and q-connectivity in wireless sensor networks, Information Networking (ICOIN), International Conference on (Bali, Indonesia 1-3 Feb. 2012) DOI: 0.1109/ICOIN.2012.6164394

\begin{tabular}{|c|c|c|c|}
\hline (c) (i) & & & \\
\hline $\begin{array}{l}\text { Journal } \\
\text { Sciences by }\end{array}$ & $\begin{array}{l}\text { of Applied } \\
\text { y BUITEMS is }\end{array}$ & $\begin{array}{l}\text { and } \\
\text { licensed }\end{array}$ & $\begin{array}{r}\text { Emerging } \\
\text { under }\end{array}$ \\
\hline a Creative & Commons & Attributio & 4.0 \\
\hline
\end{tabular}

